H. Umemura

Nagoya Math. J.

Vol. 64 (1976), 31-45

\title{
ON A CERTAIN TYPE OF VECTOR BUNDLES OVER AN ABELIAN VARIETY
}

\author{
HIROSHI UMEMURA
}

\section{Introduction}

It seems to the author that the theory of vector bundles of rank $r$ over an abelian variety of dimension $g$ is not sufficiently developed except for the two cases $r=1$ and $g=1$. The case $r=1$ is the theory of theta functions and is one of the richest branches of mathematics. It is quite natural to try to explore vector bundles in the higher rank case as we did in the case $r=1$. So far as the author knows, the following surveys on the vector bundles over an abelian variety have been done: 1. Atiyah [1] classified the vector bundles over an elliptic curve. 2. Matsushima [6] and Morimoto [8] studied the vector bundles admitting a connection over a complex torus (See also Umemura [14]). 3. Morikawa [7] and Oda [10] characterized the vector bundles isomorphic to the direct image of a line bundle under an isogeny. We can say, since the work of Atiyah [1] over an elliptic curve makes the theory of vector bundle over an elliptic curve very clear, that the theory is sufficiently developed over an elliptic curve. But as we remarked in [13], the situation becomes rather complicated if we try to proceed to the higher dimensional case. The types of the vector bundles studied by Matsushima [6], Morimoto [8], Morikawa [7] and Oda [10] are too much restricted. In fact a vector bundle admitting a connection is obtained by a representation of the fundamental group of the abelian variety and the vector bundles studied by [7] and [10] are related with theta functions. But over an elliptic curve, we have essentially only those type of vector bundles and this fact makes the theory of vector bundles over an elliptic curve simple and unfortunately very special. The aim of this paper is not try to establish a theory but to go over the framework of 1,2 and 3. To do this we propose a notion on a vector bundle, more precisely, Received October 15, 1975. 
on a Brauer-Severi scheme over an abelian variety. Namely we define a Brauer-Severi scheme of type $M$ (1.17). First of all, we remark that the vector bundles studied in 1,2 and 3 are not of type $M$. Next we show that the study of $H$-stable vector bundles over an abelian surfaces reduces to the study of vector bundles of type $M$ (Theorem 3.7). We also prove a result on the representations of the group $\mathscr{G}(E)$ (Theorem (2.9)). Our proof depends on the descent theory of Grothendieck and the theory of $H$-stable vector bundles of Takemoto [11] [12]. We use them to study how an $H$-stable vector bundle behaves when we take the direct image or the inverse image under an isogeny.

All the schemes we consider are defined over the complex number field $C$ only to avoid inseparable isogenies. So far as we work over $C$, the Zariski topology and the usual topology are sufficient but for a further purpose, it is preferable to make the theory purely algebraic. For this reason we use the étale topology instead of the usual topology.

\section{Stable Brauer-Severi schemes over an abelian variety}

Let us begin by recalling the theory of Brauer groups of schemes developed by Grothendieck [4].

Definition (1.1). Let $X$ be a scheme. A Brauer-Severi scheme over $X$ is, by definition, an $X$-scheme $P \rightarrow X$ which is, locally for the étale topology, isomorphic to the projective bundle $P_{X}^{r-1}$.

Since $\operatorname{Aut}_{k} \boldsymbol{P}^{r-1} \simeq P G L_{r}$, a Brauer-Severi scheme determines a principal $P G L_{r}$-bundle for the étale topology. Conversely if a principal $P G L_{r}$-bundle over $X$ for the étale topology is given, we can associate a Brauer-Severi scheme over $X$. Hence we get a bijection between the cohomology group $H^{1}\left(X_{\mathrm{et}}^{\prime}, P G L_{r}\right)$ and the set of isomorphism classes of Brauer-Severi schemes over $X$.

Let $P$ be a Brauer-Severi scheme over $X$. We can associate with $P$ a locally free sheaf, the Azumaya algebra of $P$ which we denote by $\mathfrak{U}(P)$ (cf. Grothendieck [4]). If $P$ is the projective bundle of a *vector bundle $E$, then $\mathscr{X}(P)=E \otimes E^{\vee}$. Conversely given an Azumaya algebra over $X$ which is, by definition, an algebra over $X$ isomorphic to the matrix algebra $M_{n}\left(\mathcal{O}_{X}\right)$ locally for the étale topology, we can associate a Brauer-Severi scheme to it.

\footnotetext{
* A vector bundle is a locally free sheaf of finite rank.
} 
The exact sequence of algebraic groups

$$
1 \rightarrow \boldsymbol{G}_{m} \rightarrow G L_{n} \rightarrow P G L_{n} \rightarrow 1
$$

gives an exact sequence of sheaves over $X_{\mathrm{et}}^{\prime}$ hence the exact sequence of cohomology groups

$$
1 \longrightarrow H^{1}\left(X_{\mathrm{e}}^{\prime}, \boldsymbol{G}_{m}\right) \longrightarrow H^{1}\left(X_{\mathrm{et}}^{\prime}, G L_{n}\right) \longrightarrow H^{1}\left(X_{\mathrm{e}}^{\prime}, P G L_{n}\right) \stackrel{\delta_{n}}{\longrightarrow} H^{2}\left(X_{\mathrm{et}}^{\prime}, \boldsymbol{G}_{m}\right) .
$$

Let $\xi$ be an element of $H^{1}\left(X_{\mathrm{e}}^{\prime}, P G L_{n}\right)$. Then a necessary and sufficient condition for lifting $\xi$ to an element of $H^{1}\left(X_{\mathrm{et}}^{\prime}, G L_{n}\right)$ i.e., to a principal $G L_{n}$-bundle is $\delta_{n}(\xi)=0$. Since $H^{1}\left(X_{\text {zar }}, G L_{n}\right) \cong H^{1}\left(X_{\text {et }}, G L_{n}\right)$, in the language of Brauer-Severi schemes, we can state this as follows: let $P$ be a Brauer-Severi scheme and $\xi$ be the corresponding principal $P G L_{n^{-}}$ bundle, then there exists a vector bundle $E$ over $X$ such that $P$ is isomorphic to $P(E)$ over $X$ if and only if $\delta_{n}(\xi)=0$. The group $\bigcup_{n \geq 1} \operatorname{Im} \delta_{n}$ is called the Brauer group of $X$ and denoted by $\mathrm{Br}(X)$. By Grothendieck [4], $\mathrm{Br}(X)$ is a torsion group (if $X$ is connected).

From now on the schemes that we consider are of finite type over an algebraically closed field $k$.

DeFinition (1.2). Let $P$ be a Brauer-Severi scheme over a scheme $X$. We say that $P$ is simple if the group scheme $\operatorname{Aut}_{X} P$ is discrete. Let $E$ be a vector bundle over $X . \quad E$ is said to be simple if the projective bundle $P(E)$ is simple.

The following lemma is well known but we prove it because our proof of the Theorems depends heavily on it.

Lemma (1.3). Let $E_{1}, E_{2}$ be vector bundles over $X$. Then the projective bundle $P\left(E_{1}\right)$ is isomorphic to $P\left(E_{2}\right)$ over $X$ if and only if there exists a line bundle $L$ over $X$ such that $E_{2}$ is isomorphic to $E_{1} \otimes L$.

Proof. Since the if part is trivial, we prove the only if part. First of all, we know that the Picard group Pic $\left(\boldsymbol{P}\left(E_{1}\right)\right)$ is isomorphic to the direct sum $Z \oplus \operatorname{Pic} X$ i.e. a line bundle over $P\left(E_{1}\right)$ is written uniquely in the form $\mathcal{O}_{\boldsymbol{P}\left(E_{1}\right)}(n) \otimes p_{1}^{*} N$ with $N \in \operatorname{Pic} X$ where $p_{1}: \boldsymbol{P}\left(E_{1}\right) \rightarrow X$ is the projection. Given an isomorphism $\varphi: P\left(E_{1}\right) \widetilde{\rightarrow}\left(E_{2}\right)$ over $X$, then there exists a line bundle $L$ over $X$ such that $\varphi * \mathcal{O}_{P\left(E_{2}\right)}(1)$ is isomorphic to $\mathcal{O}_{\boldsymbol{P}\left(E_{1}\right)}(n) \otimes p_{1}^{*} L$ for some integer $n$. Restricting the isomorphism to 
the fibre we conclude $n=1$. Hence

$$
\varphi * \mathcal{O}_{\boldsymbol{P}\left(E_{2}\right)}(1) \simeq \mathcal{O}_{\boldsymbol{P}\left(E_{1}\right)}(1) \otimes p_{1}^{*} L .
$$

Applying $p_{1^{*}}$ for (1.3.1), we get $p_{1^{*}} * \mathcal{O}_{P_{\left(E_{2}\right)}}(1) \simeq E_{1} \otimes L$. Since $p_{1^{*}} \mathcal{H}_{*} \mathcal{O}_{P\left(E_{2}\right)}(1)$ $\simeq p_{2^{*}} \mathcal{O}_{P\left(E_{2}\right)}(1)$ where $p_{2}$ is the projection $P\left(E_{2}\right) \rightarrow X$, the lemma follows.

q.e.d.

If we apply the Lemma for $E_{1}=E_{2}=E$, we obtain

COROLLARY (1.4). Let $E$ be a vector bundle over $X$. We get an exact sequence of group schemes;

$$
1 \rightarrow \operatorname{Aut}_{X} E / \Gamma\left(X, \mathcal{O}_{X}^{*}\right) \rightarrow \operatorname{Aut}_{X}(\boldsymbol{P}(E)) \rightarrow \sum(E) \rightarrow 1,
$$

where $\sum(E)$ is the group of line bundles $L$ such that $E$ is isomorphic to $E \otimes L$.

COROLLARY (1.5). If $X$ is complete and normal, then $E$ is simple if and only if $\operatorname{Hom}_{o_{X}}(E, E) \simeq k$.

Proof. Since $X$ is complete, $\Gamma\left(X, \mathcal{O}_{X}^{*}\right) \simeq k^{*}$. Moreover since $X$ is normal, $\mathrm{Pic}^{0} X$ is an abelian variety by Grothendieck [3 bis]. Since $\sum(E)$ is contained in the subgroup of rank $E$-torsion of $\operatorname{Pic}^{0} X, \sum(E)$ is discrete. Since $\mathrm{Aut}_{X} E$ is connected, Corollary (1.5) follows from the exact sequence (1.4.1).

Lemma (1.6). Let $A$ be an abelian variety and $P$ a Brauer-Severi scheme over $A$. Then, there exist an isogeny $A^{\prime} \rightarrow A$ and a vector bundle $E$ over $A^{\prime}$ such that $A^{\prime} \times{ }_{A} P$ is $A^{\prime}$-isomorphic to $P(E)$.

Proof. Let $\xi$ be an element of $H^{2}\left(A_{\text {ét }}, \boldsymbol{G}_{m}\right)$. We denote by $n_{A}$ the isogeny of $A$ onto $A$ itself sending $x \in A$ to $n x$. By Hoobler [5], $n_{A}^{*} \xi$ $=n^{2} \xi+\left(\frac{n^{2}-n}{2}\right)\left(\left(-1_{A}\right) *(\xi)-\xi\right)$. As we remarked above, $\operatorname{Br}(X)$ is torsion. Hence if $\xi \in B r(X)$, there exists a positive integer $n$ such that $n_{A}^{*} \xi=0$. This shows that there exists an integer $n \geq 0$ such that $n_{A}^{*} P$ $=A^{\prime} \times{ }_{A} P$ is $A^{\prime}$-isomorphic to $P(E)$ for a vector bundle $E$ over $A$.

Remark (1.6.1). We can prove Lemma (1.6) by using the Künneth formula, which seems more natural.

We shall study the stable vector bundles in the sence of Takemoto 
[11]. Our stability is more restrictive than the stability recently introduced by Gieseker [2].

DEFINITION (1.7). Let $V$ be a nonsingular projective surface. Let $H$ be an ample line bundle over $V$. Let $E$ be a vector bundle i.e., a locally free sheaf of finite rank over $V$. We say that $E$ is $H$-stable (resp. $H$-semi-stable) if for any subsheaf $F \subset E$ with $0 \neq r(F) \neq r(E)$, we have

$$
\begin{gathered}
\frac{\left(H \cdot C_{1}(F)\right)}{r(F)}<\frac{\left(H \cdot C_{1}(E)\right)}{r(E)}, \text { where }(\cdot) \\
(\text { resp. } \leq)
\end{gathered}
$$

denotes the intersection number, $C_{1}(\quad)$ is the first Chern class and $r($ ) is the rank of the torsion free sheaf. For basic properties of stable bundles, see Takemoto [11]. However we remark here that if $L$ is a line bundle, then $E$ is $H$-stable (resp. $H$-semi-stable) if and only if $E \otimes L$ is $H$-stable (resp. $H$-semistable).

Definition (1.8). Let $A$ be an abelian variety and $P$ a BrauerSeveri scheme over $A . P$ is said to be absolutely simple if, for any isogeny $A^{\prime} \rightarrow A, A^{\prime} \times{ }_{A} P$ is simple. A vector bundle $E$ is said to be absolutely simple if $P(E)$ is absolutely simple.

EXAMPLE (1.9). A line bundle is absolutely simple. The direct image of a line bundle under an isogeny is not absolutely simple. Hence by the classification of Atiyah [1], a vector bundle over an elliptic curve is not absolutely simple unless its rank is 1 . If $A$ is an abelian surface, an $H$-stable bundle of rank $p$ with $(p-1) C(E)^{2}-2 p C_{2}(E) \neq 0$ is absolutely simple where $p$ is a prime number (cf. Theorem 3.7). See also example (3.12).

Definition (1.10). Let $A$ be an abelian surface. Let $H$ be an ample line bundle and $P$ a Brauer-Severi scheme over $A$. We say that $P$ is $H$-stable (resp. $H$-semi-stable) if there exist an isogeny $\varphi: A^{\prime} \rightarrow A$ and a vector bundle $E^{\prime}$ over $A^{\prime}$ such that $A^{\prime} \times_{A} P$ is $A^{\prime}$-isomorphic to $P\left(E^{\prime}\right)$ and such that $E^{\prime}$ is $\varphi * H$-stable (resp. $\varphi * H$-semi-stable)

Our definition of the stability heavily depends on the line bundle $H$. But we often omit $H$ when no confusion is possible.

From now on all the schemes we consider are defined over the complex number field $\boldsymbol{C}$. 
Let $X$ be a nonsingular projective surface. Let $\varphi: Y \rightarrow X$ be a finite Galois covering of $X$ with Galois group $G$. We recall how stable bundles behave under $\varphi^{*}$ and $\varphi_{*}$.

Proposition (1.11) (Takemoto [12]). Let $H$ be an ample line bundle over $X$ and $E$ an $H$-stable vector bundle. We assume that $G$ is solvable. Then, (i) $\varphi^{*} E$ is $\varphi^{*} H$-semi-stable, (ii) there exist a $\varphi^{*} H$-stable vector bundle $F$ over $Y$ and a subset $S \subset G$ such that $\varphi^{*} E$ is isomorphic to the direct sum $\underset{x \in S}{\oplus} T_{x}^{*} F$, where $T_{x}$ is the translation by $x$.

Proposition (1.12) (Takemoto [12]). Let $E$ be an $\varphi^{*} H$-stable vector bundle over $Y$ for an ample line bundle $H$ over $X$. If the Galois group $G$ is cyclic and of prime order, and if $E$ is not isomorphic to $\varphi^{*} F$ for any vector bundle $F$ over $X$, then the direct image $\varphi_{*} E$ is $H$-stable.

PROPOSITIPN (1.13) (Takemoto [12]). Let E be a simple vector bundle over a non-singular and complete scheme $X$. If there exists a nontrivial line bundle $L$ over $X$ such that $E$ is isomorphic to $E \otimes L$, then there exist a non-trivial unramified cyclic covering $\varphi: Y \rightarrow X$ and $a$ vector bundle $F$ over $Y$ such that $\varphi_{*} F$ is isomorphic $E$.

Let $A$ be an abelian variety and $P$ a Brauer-Severi scheme over $A$. Consider the set $H(P)$ of all the (closed) points $x \in A$ such that $P$ is isomorphic to $T_{x}^{*} P$ where $T_{x}$ is the translation by $x$. It is obvious that $H(P)$ is a subgroup of $A$. Let $\mathscr{G}(P)$ be the set of pairs $(x, \varphi)$ such that $x \in H(P)$ and $\varphi$ is an isomorphism of $P$ to $T_{x}^{*} P$. Now we make $\mathscr{G}(P)$ into a group. Let $(x, \varphi),(y, \psi)$ be elements of $\mathscr{G}(P)$. We consider the composition $T_{x}^{*} \psi \circ \varphi: P \stackrel{\varphi}{\longrightarrow} T_{x}^{*} P \stackrel{T_{x}^{*} \psi}{\longrightarrow} T_{x}^{*} T_{y}^{*} P=T_{x+y}^{*} P$. Define $(y, \psi) \circ(x, \varphi)$ $=\left(x+y, T_{x}^{*} \psi \circ \varphi\right)$. It is easy to check that $\mathscr{G}(P)$ is a group under this composition law $\circ$.

Proposition (1.14). $H(P)$ and $\mathscr{G}(P)$ are algebraic groups. $H(P)$ is a closed subgroup of $A$. We have an exact sequence;

$$
1 \longrightarrow \operatorname{Aut}_{A} P \longrightarrow \mathscr{G}(P) \stackrel{\pi}{\longrightarrow} H(P) \longrightarrow 0,
$$

where $\pi$ is defined by $(x, \varphi) \mapsto x$.

Proof. The proof is the same as that of H. Umemura [14]. Hence we omit it. 
Lemma (1.15). Let $E$ be a vector bundle over $A$. Then, $H(P(E))$ $=\{x \in A \mid$ there exists a line bundle $L$ (depending on the point $x$ ) over $A$ such that $\left.E \simeq L \otimes T_{x}^{*} E\right\}$.

Proof. This is an immediate consequence of Lemma (1.3).

Let $K$ be a finite subgroup of $H(P)$. Let $\tilde{K}$ be a subgroup of $\mathscr{G}(P)$ such that $\tilde{K}$ is mapped isomorphically to $K$ by the projection $\pi$, namely we are given a section of $\pi$ over $K$. This is equivalent to giving an action of the group $K$ on $P$ covering its action on $A$ hence also equivalent to giving a descent data for the isogeny $\varphi: A \rightarrow A / K$. In this case, by the descent theory of Grothendieck [3], $P$ descends through the isogeny $\varphi: A \rightarrow A / K$. Conversely, if there exists a Brauer-Severi scheme $P$ over $A / K$ such $A \times_{A / K} P^{\prime}$ is $A$-isomorphic to $P$, then we can find an action $K$ on $P$. Hence there is a $1-1$ correspondence between the set of subgroups of $\mathscr{G}(P)$ which are mapped isomorphically onto $K$ and the set of isomorphism classes of Brauer-Severi schemes over $A / K$ of which the pull-back over $A$ is isomorphic to $P$.

Let $P: A_{1} \rightarrow A_{2}$ be an isogeny of abelian varieties. Let $K$ be the kernel of $\varphi$. Let $P_{i}$ be a Brauer-Severi scheme over $A_{i}, i=1,2$. Suppose $P_{2} \times{ }_{A_{2}} A_{1}$ is isomorphic to $P_{1}$. In this situation let us compare $\mathscr{G}\left(\boldsymbol{P}_{1}\right)$ with $\mathscr{G}\left(\boldsymbol{P}_{2}\right)$. Let $\tilde{K}$ be the subgroup of $\mathscr{G}\left(\boldsymbol{P}_{1}\right)$ lying over $K$ and corresponding to $P_{2}$. Let $(y, \psi) \in \mathscr{G}\left(P_{1}\right)$. By the descent theory of Grothendieck, the isomorphism $\psi: P \simeq T_{y}^{*} P$ descends through $K$ if and only if it commutes with the descent data, namely the diagram;

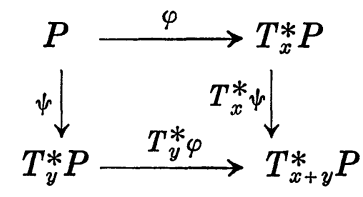

commutes for all $(x, \varphi) \in \tilde{K}$. In other words $(y, \psi)$ belongs to the centralizer of $\tilde{K}$. Hence we conclude that $\mathscr{G}\left(P_{2}\right)$ is isomorphic to the quotient group (the centralizer of $\tilde{K}$ ) $/ K$.

Definition (1.16). We write $(P, A)$ for a Brauer-Severi scheme $P$ over an abelian variety $A$. Let $\left(P^{\prime}, A^{\prime}\right)$ be another such pair. We denote $(P, A) \geq\left(P^{\prime}, A^{\prime}\right)$ if there exists an isogeny $\varphi: A \rightarrow A^{\prime}$ such that $A \times{ }_{A^{\prime}} P^{\prime}$ is $A$-isomorphic to $P$. Then the relation $\geq$ defines an order in the set of pairs $(P, A)$. 
Definition (1.17). A Brauer-Severi scheme $P$ over an abelian variety $A$ is said to be of type $M$ if the following condition is satisfied; for any pair $\left(P^{\prime}, A^{\prime}\right) \geq(P, A)$ the set $\left\{\left(P^{\prime \prime}, A^{\prime \prime}\right) \mid\left(P^{\prime}, A^{\prime}\right) \geq\left(P^{\prime \prime}, A^{\prime \prime}\right)\right\}$ has the unique minimal element.

When a Brauer-Severi scheme $P$ over $A$ is of type $M$, the unique minimal element is called the minimal model of $(P, A)$.

A Brauer-Severi scheme $P$ over an abelian variety $A$ is said to be of type $M_{0}$ if $(P, A)$ is of type $M$ and if its minimal model is isomorphic to the projective bundle of a vector bundle.

A vector bundle $E$ over an abelian variety $A$ is said to be of type $M$ (resp. $\left.M_{0}\right)$, if $(P(E), A)$ is if type $M$ (resp. $\left.M_{0}\right)$.

\section{§2. Representations of $\mathscr{G}(E)$}

Let $A$ be an abelian variety and $E$ a vector bundle over $A$. We define groups $\mathscr{G}(E)$ and $H(E)$ as in Umemura [13]. Let us briefly recall the definition. $H(E)=\left\{x \in A \mid E \simeq T_{x}^{*} E\right\}$ and $\mathscr{G}(E)$ is the set of all pairs $(x, \varphi)$ such that $x \in H(E)$ and such that $\varphi$ is an isomorphism of $E$ and $T_{x}^{*} E$. The composition law is defined similarly as in the case of Brauer-Severi schemes. Then we have a natural exact sequence of algebraic groups

$$
1 \rightarrow \operatorname{Aut} E \rightarrow \mathscr{G}(E) \rightarrow H(E) \rightarrow 0
$$

where the morphism $\mathscr{G}(E) \rightarrow H(E)$ is given by $(x, \varphi) \mapsto x$.

If $E$ is simple, the exact sequence (2.1) reduces to the sequence

$$
1 \rightarrow k^{*} \rightarrow \mathscr{G}(E) \rightarrow H(E) \rightarrow 0 .
$$

Later we shall mainly concern with this situation.

To study the group $\mathscr{G}(E)$, we had better work in a more general setting. Let $H$ be an abelian group written additively. Let $G$ be a group such that we have an exact sequence

$$
1 \rightarrow k^{*} \rightarrow G \rightarrow H \rightarrow 0
$$

and such that $k^{*}$ is contained in the center of $G$. Under the assumption that $H$ is finite, we shall determine all the finite dimensional irreducible representations of $G$ on which $k^{*}$ acts naturally.

DEFinition (2.3). A level subgroup $\tilde{K}$ of $G$ is a subgroup of $G$ such that $\tilde{K} \cap k^{*}=1$ i.e., $\tilde{K}$ is isomorphic to its image $K$ in $H$. A 
maximal level subgroup is a level subgroup which is maximal among the level subgroups.

Let $x, y \in H$ and $\tilde{x}, \tilde{y}$ be elements of $G$ lying respectively over $x$ and $y$. If we put $(x, y)=\tilde{x}^{-1} \tilde{y}^{-1} \tilde{x} \tilde{y}$, then $(x, y) \in k^{*},(x, y)$ is independent of the choice of $\tilde{x}$ and $\tilde{y}$ hence $(x, y)$ defines a bilinear form on $H$. Let $K$ be a subgroup of $H$. Then there exists a level subgroup $\tilde{K}$ lying over $K$ if and only if $(x, y)=1$ for any $x, y \in K$. Since $k$ is algebraically closed, for any cyclic subgroup $K$ of $H$, there exists a level subgroup $\tilde{K}$ lying over $K$. In the geometric case $G=\mathscr{G}(E), H=H(E)$, a level subgroup $\tilde{K}$ lying over a closed subgroup $K \subset H(E)$ corresponds to giving a descent data for $E$ with respect to the natural projection $A \rightarrow$ $A / K$. Hence by the descent theory of Grothendieck [3], $E$ descends through the natural projection $A \rightarrow A / K$ if there exists a level subgroup lying over $K$ or if the restriction of the bilinear form (, ) to $K$ is trivial. Let $\tilde{K} \subset \mathscr{G}(E)$ be a level subgroup lying over $K$ which we assume to be closed. Hence $E$ descends through the natural projection $A \rightarrow A / K$ and gives a vector bundle $F$ over $A / K$. The descent theory of Grothendieck [3] teaches us $(x, \varphi) \in \mathscr{G}(E)$ comes from $\mathscr{G}(F)$ if and only if $(x, \varphi)$ commutes with the descent data for $E$ i.e., $(x, \varphi)$ is in the centralizer of $\tilde{K}$. Hence $\mathscr{G}(F)$ is isomorphic to the quotient group (the centralizer of $\tilde{K}) / K$.

From now on we assume that $H$ is finite, all the representations are finite dimensional and that the group $k^{*}$ acts naturally on them. Then, the argument in the proof of Proposition 3 in Mumford: On the equations defining abelian varieties I, Invent. math. 1, 287-354 (1966), gives the following lemma and proposition.

LEMMA (2.4). The group $G$ is completely reducible.

Proposition (2.5). Let $V$ be an irreducible representation of $G$. Let $\tilde{K}$ be a maximal level subgroup and $K$ its image in $H$. Then $\operatorname{dim}_{k} V$ $=[H ; K]$. Let $V^{\prime}$ be another irreducible representation of $G$. If $V$ and $V^{\prime}$ a are isomorphic as representations of $\tilde{K}$, then they are isomorphic as representations of $G$.

From the proposition we deduce easily,

COROLlaRY (2.6). The groups $G$ is abelian if and only if $G$ has a representation of degree 1. 
CoRollary (2.7). Let $V=\underset{x \in \operatorname{Hom}\left(\tilde{K}, k^{*}\right)}{\bigoplus_{x}} V_{x}, V^{\prime}=\underset{x^{\prime} \in \operatorname{Hom}\left(\tilde{K}, k^{*}\right)}{\bigoplus_{x^{\prime}}} V^{\prime}$ be two irreducible representations of $G$. If there exists $\chi=\chi^{\prime}$ such that the eigenspaces $V_{x}$ and $V_{x^{\prime}}^{\prime}$ are not zero, then $V$ and $V^{\prime}$ are equivalent representations.

CoRollaRY (2.8). Let $V=\underset{x \in \operatorname{Hom}\left(\tilde{K}, k^{*}\right)}{\bigoplus_{x}} V_{x}$ be a representation of $G$. Suppose that the eigenspace $V_{x}$ is not zero for any character $\chi$. T.hen $V$ contains all the irreducible representations of $G$.

THEOREM (2.9). Let $E$ be a vector bundle over an abelian variety $A$. If $E$ is simple and the Euler-Poincaré characteristic $\chi(E)$ is not zero, then there exists an $i$ such that the cohomology group $H^{i}(A, E)$ contains every irreducible representation of the group $\mathscr{G}(E)$.

Proof. First of all we show that the group $H(E)$ is finite. In fact $H(E)$ is contained in $\chi(E)$-torsion subgroup of $A$. For, assume that $H(E)$ is not finite, then there exists an $n$-torsion element for any integer $n \geq 1$. But if $x$ is $n$-torsion, the extension (2.2) splits over the cyclic group $(x)$ since $k$ is algebraically closed. This shows that $E$ descends through the isogeny $A \rightarrow A /(x)$. Therefore $n$ devides $\chi(E)$. Let $\tilde{K}$ be a maximal level subgroup and $K$ its image in $H(E)$. By the descent theory, there exists a vector bundle $E^{\prime}$ over $A^{\prime}=A / K$ such that $E \simeq \varphi^{*} E^{\prime}$ where $\varphi$ is the natural isogeny of $A$ onto $A^{\prime}$. Consider the vector bundle $p_{1}^{*} E^{\prime} \otimes \mathscr{P}$ over $A^{\prime} \times \check{A}^{\prime}$ where $p_{1}$ is the first projection, $\check{A}^{\prime}$ is the dual abelian variety of $A^{\prime}$ and $\mathscr{P}$ is the Poincare line bundle. Since $\chi\left(E^{\prime}\right) \neq 0$, $\chi\left(E^{\prime} \otimes L\right) \neq 0$ for any $L \in \operatorname{Pic}^{0} A^{\prime}$. Hence there exist an $i$ such that $H^{i}\left(A, E^{\prime} \otimes L\right) \neq 0$ for the generic line bundle $L \in \mathrm{Pic}^{0} A^{\prime}$ hence, by the upper semi-continuity for any $L \in \mathrm{Pic}^{0} A^{\prime}$. Now we notice the decomposition

$$
H^{i}(A, E)=H^{i}\left(A, \varphi^{*} E^{\prime}\right) \simeq \bigoplus_{\varphi^{*} \overbrace{\simeq O_{A}}} H^{i}\left(A^{\prime}, E^{\prime} \otimes L\right)
$$

On the cohomology group $H^{i}\left(A^{\prime}, E^{\prime} \otimes L\right)$ the kernel $K$ acts by the character $\chi$ where $\chi$ is the character of $K$ defining $L$. Since in the decomposition $\varphi_{*} \mathcal{O}_{A} \simeq \bigoplus_{\varphi^{*} L \simeq \mathcal{O}_{A}} L$ every character of $K$ appears (cf. Mumford [9]), every character of the maximal level subgroup $\tilde{K}$ appears in $H^{i}(A, E)$. Hence it contains all the irreducible representation of $\mathscr{G}(E)$ by Corollary (2.8).

q.e.d. 


\section{§3. Vector bundles of type $M$ and stable vector bundles}

LEMMA (3.1). Let $A$ be an abelian variety and $P$ a Brauer-Severi scheme over $A$. If $P$ is absolutely simple, then Aut $_{A^{\prime}} P^{\prime}$ consists of a single element for any $\left(P^{\prime}, A^{\prime}\right) \geq(P, A)$.

Proof. It is sufficient to show that if $E$ is a vector bundle over $A^{\prime}$ such that $\left(P(E), A^{\prime}\right) \geq(P, A)$, then $\mathrm{Aut}_{A^{\prime}} P(E)$ consists of a single element. To show this, by exact sequence (1.4.1), we have to prove $\sum(E)=\left\{\mathcal{O}_{A^{\prime}}\right\}$. If $L \in \sum(E)$ and $L \neq \mathcal{O}_{A^{\prime}}$, then by Proposition (1.13), $E$ would be a direct image of a vector bundle of smaller rank. This contradicts the assumption that $P$ is absolutely simple.

LEMMA (3.2). Let $A$ be an abelian variety and $P$ a Brauer-Severi scheme over $A$. If $P$ is absolutely simple and $H(P)$ is finite, then $P$ is of type $M$.

Proof. By the descent theory in $\S 1, H\left(P^{\prime}\right)$ is finite for any $\left(P^{\prime}, A^{\prime}\right)$ $\geq(P, A)$. Since Aut $_{A^{\prime}} P^{\prime}=\{1\}$, the set of descent data corresponds to the set of subgroups $H\left(P^{\prime}\right)$.

COROLlary (3.3). Let $P$ be an absolutely simple Brauer-Severi scheme over an abelian variety $A$. If the Euler-Poincaré characteristic $\chi(\Re(P))$ is not zero, then $P$ is of type $M$.

Proof. Let $(P, A) \geq\left(P^{\prime}, A^{\prime}\right)$. Then $(\mathfrak{H}(P), A) \geq\left(\mathfrak{H}\left(P^{\prime}\right), A^{\prime}\right)$. Hence $\chi\left(\mathfrak{Y}\left(P^{\prime}\right)\right)$ devides $\chi(\mathscr{Y}(P))$. Therefore $H(P)$ is finite.

CoRollary (3.4). Let $P$ be an absolutely simple Brauer-Severi scheme over an abelian variety $A$. If $H(P)=\{0\}$, then $P$ is of type $M$ and minimal.

LEMMA (3.5). Let $E$ be an absolutely simple vector bundle of type $M$ over an abelian variety $A$. If $\chi(\operatorname{det} E)$ is prime to the rank $r$ of $E$, then $E$ is of type $M_{0}$.

Proof. It follows from the assumption that $H(P(E))$ is finite. Hence there exists $\left(E^{\prime}, A^{\prime}\right)$ such that $(P(E), A) \geq\left(P\left(E^{\prime}\right), A^{\prime}\right)$ and such that for any line bundle $M$ over $A^{\prime}, E^{\prime} \otimes M$ does not descends through any isogeny of degree $\geq 2$ i.e., $H\left(E^{\prime} \otimes M\right)=\{0\}$. We shall show $H\left(P\left(E^{\prime}\right)\right.$ ) $=\{0\}$. Let $x \in H\left(P\left(E^{\prime}\right)\right)$ be an $r$-division point. Then by Lemma (1.15), there exists a line bundle $L$ such that $T_{x}^{*} E^{\prime} \simeq E^{\prime} \otimes L$. Hence $T_{x}^{*} \operatorname{det} E^{\prime}$ 
$\simeq \operatorname{det} E^{\prime} \otimes L^{\otimes r} . \quad$ Since $r x=0, \quad E^{\prime} \simeq T_{r x}^{*} E^{\prime} \simeq E^{\prime} \otimes L^{\otimes r} . \quad$ By Proposition (1.13), this implies $L^{\otimes r} \simeq \mathcal{O}_{A^{\prime}}$ because $E^{\prime}$ is absolutely simple. Therefore $T_{x}^{*} \operatorname{det} E^{\prime} \simeq \operatorname{det} E^{\prime}$. Since $\chi\left(\operatorname{det} E^{\prime}\right)$ divides $\chi(\operatorname{det} E), \chi\left(\operatorname{det} E^{\prime}\right)$ is prime to $r$. Since $\left|H\left(\operatorname{det} E^{\prime}\right)\right|=\chi\left(\operatorname{det} E^{\prime}\right)^{2}$, the order of the group $H\left(\operatorname{det} E^{\prime}\right)$ is prime to $r$. Hence $x=0$. Let $0 \neq x \in H\left(P\left(E^{\prime}\right)\right)$, then the order of $x$ is prime to $r$. As above there exists a line bundle $L \neq \mathcal{O}_{A^{\prime}}$ such that $T_{x}^{*} E^{\prime} \simeq E^{\prime} \otimes L$. Taking the determinant,

$$
T_{x}^{*} \operatorname{det} E^{\prime} \simeq \operatorname{det} E^{\prime} \otimes L^{\otimes r} .
$$

Since the order $\ell$ of $x$ is prime to $r$, the order of $L \in \operatorname{Pic}^{0} A^{\prime}$ is prime to $r$. Hence there exist integers $a, b$ such that $a l+b r=1$. From (3.6), we get $T_{x}^{*}\left(\operatorname{det} E^{\prime}\right)^{\otimes b} \simeq\left(\operatorname{det} E^{\prime}\right)^{\otimes b} \otimes L^{\otimes r b} \simeq\left(\operatorname{det} E^{\prime}\right)^{\otimes b} \otimes L$. Hence if we put $M=\left(\operatorname{det} E^{\prime}\right)^{\otimes-b}$, then $T_{x}^{*} M \simeq M \otimes L^{-1}$. If we consider $E^{\prime} \otimes M$, then $T_{x}^{*}\left(E^{\prime} \otimes M\right) \simeq T_{x}^{*} E^{\prime} \otimes T_{x}^{*} M \simeq\left(E^{\prime} \otimes L\right) \otimes\left(M \otimes L^{-1}\right) \simeq E^{\prime} \otimes M . \quad$ Therefore $H\left(E^{\prime} \otimes M\right) \neq\{0\}$. This contradicts the definition of $E^{\prime}$.

THEOREM (3.7). Let $E$ be a vector bundle of rank $r$ and $H$ an ample line bundle over an abelian surface $A$. We assume that $E$ is $H$ stable.

(1) If $(r-1) C_{1}(E)^{2}-2 r C_{2}(E)=0$, then $E$ is the direct image of a line bundle under an isogeny.

(2) If $(r-1) C_{1}(E)^{2}-2 r C_{2}(E) \neq 0$, then $E$ is the direct image of an absolutely simple vector bundle of type $M$ under an isogeny.

(3) If $E$ is absolutely simple and of type $M$ and if the rank $r$ is prime to the Euler-Poincaré characteristic $\chi(\operatorname{det} E)$, then $E$ is of type $M_{0}$.

Proof. The assertion (1) is well-known and proved by Morikawa [7] and Oda [10]. Another proof is given in Umemura [15]. If there exists $\left(E^{\prime}, A^{\prime}\right) \geq(E, A)$ such that $E^{\prime}$ is the direct image of a stable vector bundle under an isogeny of degree $\geq 2$, then $E$ is also the direct image of a stable vector bundle under an isogeny of degree 2 . In fact, since $E^{\prime}$ is the direct image under an isogeny of degree $\geq 2$, there exists a non-trivial line bundle $L^{\prime}$ over $A^{\prime}$ such that $E^{\prime} \simeq E^{\prime} \otimes L^{\prime}$. Since $L^{\prime}$ is algebraically equivalent to 0 , there exists a line bundle $L$ over $A$ with $L^{\prime}=\varphi^{*} L$ where $\varphi$ is the isogeny giving $\left(E^{\prime}, A^{\prime}\right) \geq(E, A)$. Then $\varphi^{*} E \simeq$ $\varphi^{*} E \otimes L^{\prime} \simeq \varphi^{*}(E \otimes L)$. Therefore, taking the direct image $\varphi_{*}, E \simeq E \otimes L^{\prime \prime}$ with a non-trivial line bundle $L^{\prime \prime}$ over $A$. Hence by Proposition (1.13) $E$ is the direct image of a stable vector bundle $F$ under an isogeny of 
degree $\geq 2$. We notice that so far we used only the stability of $E$. If $F$ is not absolutely simple, repeating this process, we can prove that $E$ is the direct image under an isogeny of a stable vector bundle which is absolutely simple. Thus we may assume that there exists an isogeny $\varphi: A^{\prime}: \rightarrow A$ and an absolutely simple vector bundle $F$ over $A^{\prime}$ such that $\varphi_{*} F \simeq E$. If we notice that $-(r-1) c_{1}(E)^{2}+2 r c_{2}(E)$ is the second Chern class of the Azumaya algebra $E \otimes \check{E}$ of $P(E)$, it is easy to see $-\left(r^{\prime}-\right.$ 1) $c_{1}(F)^{2}+2 r^{\prime} c_{2}(F) \neq 0$ where $r^{\prime}$ is the rank of $F$. Corollary (3.3) now shows that $F$ is of type $M$. Hence the assertion (2) is proved. The last assertion is nothing but Lemma (3.5).

We define the relation $\geq$ for vector bundles over abelian varieties as we did for Brauer-Severi schemes.

DeFinition (3.8). We write $(E, A)$ for a vector bundle $E$ over an abelian variety $A$. Let $\left(E^{\prime}, A^{\prime}\right)$ be another such pair. We denote $(E, A)$ $\geq\left(E^{\prime}, A^{\prime}\right)$ if there exists an isogeny $\varphi: A \rightarrow A^{\prime}$ such that the inverse image $\varphi^{*} E^{\prime}$ is isomorphic to $E$.

DEFinition (3.9). Let $E$ be a vector bundle over an abelian variety $A$. If $E$ is of type $M_{0}$ and if $P(E)$ is the minimal model, then we say that $E$ is minimal.

DEFINITION (3.10). Let $E$ be a vector bundle over an abelian variety $A$. If $E$ is of type $M_{0}$ and if there exists $\left(E^{\prime}, A^{\prime}\right) \geq\left(E^{\prime}, A^{\prime}\right)$ and $E^{\prime}$ is minimal, then we say that $E$ is normalized.

THEOREM (3.11). Let $E$ be a vector bundle over an abelian variety A. If $E$ is absolutely simple, then the following are equivalent;

(1) $E$ is of type $M_{0}$ and normalized.

(2) $\mathscr{G}\left(E^{\prime}\right)$ is abelian and $H\left(E^{\prime}\right)$ is finite if we have either $\left(E^{\prime}, A^{\prime}\right) \geq(E, A)$ or $(E, A) \geq\left(E^{\prime}, A^{\prime}\right)$.

(3) $\mathscr{G}\left(E^{\prime}\right)$ is abelian and $H\left(E^{\prime}\right)$ is finite if we have $\left(E^{\prime}, A^{\prime}\right) \geq(E, A)$.

Proof. Let $E^{\prime \prime}$ be a vector bundle over an abelian variety $A^{\prime \prime}$. Assume that $H\left(E^{\prime \prime}\right)$ is finite and that $\mathscr{G}\left(E^{\prime \prime}\right)$ is not abelian. Then there exist two maximal level subgroups $\tilde{K}_{1}$ and $\tilde{K}_{2}$ lying respectively over $K_{1}$ and $K_{2}$ with $K_{1} \neq K_{2}$. This gives vector bundles $E_{1}$ and $E_{2}$ such that $\left(E^{\prime \prime}, A^{\prime \prime}\right) \geq\left(E_{i}, A^{\prime \prime} / K_{i}\right) i=1,2$. By the maximality of $\tilde{K}_{i}, E_{i}$ descends no more. We now show that (1) implies (3). By the observation above, 
it is sufficient to show that $H\left(E^{\prime}\right)$ is finite. If $H\left(E^{\prime}\right)$ is not finite, then the algebraic group $H\left(P\left(E^{\prime}\right)\right.$ ) contains an element $x$ of order $n$ for any integer $n \geq 1$. Hence $P\left(E^{\prime}\right)$ descends through the isogeny $A \rightarrow A /(x)$ which is of degree $n$. Therefore $H\left(E^{\prime}\right)$ is finite. Since (3) is a special case of (2), (2) $\Rightarrow(3)$. By the descent theory (3) implies (2). We have to show that (3) implies (1). Let $\left(E^{\prime}, A^{\prime}\right) \geq\left(E^{\prime \prime}, A^{\prime \prime}\right)$ and we assume that $E^{\prime \prime}$ corresponds to a maximal level subgroup. Since $\mathscr{G}\left(E^{\prime}\right)$ is abelian, $A^{\prime \prime} \simeq A^{\prime} / H\left(E^{\prime}\right)$.

\section{LEMMA (3.12) $H\left(P\left(E^{\prime \prime}\right)\right)=0$.}

Proof of Lemma (3.12). We assume $H\left(P\left(E^{\prime \prime}\right)\right) \neq 0$ and show that this gives a contradiction. Since $H\left(P\left(E^{\prime \prime}\right)\right) \neq 0$ is a closed subgroup of the abelian variety $A$, it contains an element $x \neq 0$ of finite order. Therefore there exists a line bundle $L$ such that $E^{\prime \prime} \simeq T_{x}^{*} E^{\prime \prime} \otimes L$ by Lemma (1.3). Since $E^{\prime \prime}$ does not descend any more, $L \neq \mathcal{O}_{A^{\prime \prime}}$. Since $L$ is a tortion element of $\operatorname{Pic}^{0}\left(A^{\prime \prime}\right)$, we can find an isogeny $\varphi: A^{(3)} \rightarrow A$ such that $\varphi^{*} \psi^{*} L \simeq \mathcal{O}_{A(3)}$ where $\psi$ is the natural isogeny $A^{\prime} \rightarrow A / K=A^{\prime \prime}$. Consider the inverse image $E^{(3)}=\varphi^{*} \psi^{*} E^{\prime \prime}$, then $H\left(E^{\prime \prime}\right) \nsupseteq \operatorname{Ker} \varphi \circ \psi$. But there is a maximal level subgroup lying over $\operatorname{Ker} \varphi \circ \psi$ since $E^{\prime \prime}$ descends no more. This shows that $\mathscr{G}\left(E^{(3)}\right)$ is not abelian. This is a contradiction, since $\left(E^{(3)}, A^{(3)}\right) \geq(E, A)$.

q.e.d.

Now by Corollary (3.4), $E$ is of type $M$ and $E^{\prime \prime}$ is minimal.

COROLlaRY (3.13). If $E$ is of type $M_{0}$ and normalized, then $\mathscr{G}(E)$ $=k^{*} \times H(E)$.

COROLLARY (3.14). Let $E$ be a vector bvndle over an abelian variety A. If $E$ is absolutely simple, $H(P(E)$ ) is finite and if there exists an integer $i \geq 0$ such that $\operatorname{dim} H^{i}\left(A^{\prime}, E^{\prime}\right)=1$ for any $\left(E^{\prime}, A^{\prime}\right) \geq(E, A)$, then $E$ is of type $M_{0}$ and normalized.

Proof. It follows from Corollary (2.6) that $\mathscr{G}\left(E^{\prime}\right)$ is abelian. The assertion now follows from Theorem (3.11).

EXAMPLE (3.15). Let $A$ be an abelian variety of dimension $g$. We construct a vector bundle of type $M_{0}$ over $A$. Let $L$ be a very ample line bundle over $A$. Let $\varphi_{1}, \varphi_{2}, \cdots, \varphi_{g+1}$ be linearly independent sections of $L$ which do not vanish simultaneously. Let us define $E$ by the exact sequence ; 


$$
\begin{gathered}
0 \rightarrow \mathcal{O} \rightarrow L^{\oplus g+1} \rightarrow E \rightarrow 0 . \\
1 \mapsto\left(\varphi_{1}, \varphi_{2}, \cdots, \varphi_{g+1}\right)
\end{gathered}
$$

By the same argument as in Umemura [13], $E$ is simple. The inverse image of $E^{\prime}$ under an isogeny is again defined by the same type of exact sequence. Hence $E$ is absolutely simple. $H(E)$ is a subgroup of $H(\operatorname{det} E)$ which is finite because $\operatorname{det} E$ is ample. $H(E)$ is a subgroup of $H(P(E))$. But those two groups coincide. In fact, suppose $E \simeq T_{x}^{*} E \otimes M$ for a line bundle $M$, then from the exact sequence defining $E$, we conclude $H^{g-1}(A, E)=H^{g}(A, \mathcal{O}) \simeq k, H^{g-1}\left(A, T_{x}^{*} E \otimes M\right)=0$ if $M$ is not trivial. Hence $M$ should be trivial. Therefore $H(P(E))$ is finite. By Lemma (3.2) and Corollary (3.14), $E$ is of type $M_{0}$ and normalized.

\section{REFERENCES}

[1] Atiyah, M. F. Complex analytic connections in fibre bundles, Trans. Amer. Math. Soc., 85 (1957), 181-207.

[2] Gieseker, D. On the moduli of vector bundles on an algebraic surface.

[ 3 ] Grothendieck, A. Technique de descente et théorème d'existence en géométrie algébrique, I. Séminaire Bourbaki, $12(1959 / 60), n^{\circ} 190$.

[ 3 bis] _ Technique de descente et théorèmes d'existence en géométrie algébrique VI, Les schemas de Picard: Propriétés générales, Séminaire Bourbaki, 14 (1961/ $62), n^{\circ} 236$.

[4] Grothendieck, A. Le groupe de Brauer I, Séminaire Bourbaki, $17(1964 / 65), \mathrm{n}^{\circ}$ 290.

[ 5 ] Hoobler, R. Brauer groups of abelian schemes, Ann. Scient. Ec. Norm. Sup., $4^{\mathrm{e}}$ série, t. 5 (1972), 45-70.

[6] Matsushima, Y. Fibrés holomorphes sur un tore complexe, Nagoya Math. J. 14 (1959), 1-24.

[ 7 ] Morikawa, H. A note on holomorphic vector bundles over complex tori, Nagoya Math. J., 41 (1971), 101-106.

[ 8 ] Morimoto, A. Sur la classification des espaces fibrés vectoriels holomorphes sur un tore complexe admettant des connections holomorphes, Nagoya Math. J., 15 (1959), 83-154.

[9] Mumford, D. Abelian varieties, Oxford University Press (1970), London.

[10] Oda, T. Vector bundles on an elliptic curve, Nagoya Math. J., 43 (1971), 41-72.

[11] Takemoto, F. Stable vector bundles on algebraic surfaces, Nagoya Math. J., 47 (1972), 29-48.

[12] Stable vector bundles on algebraic surfaces II, Nagoya Math. J., 52 (1973), 173-195.

[13] Umemura, H. Some results in the theory of vector bundles, Nagoya Math. J., 52 (1973), 97-128.

[14] — A theorem of Matsushima, Nagoya Math. J., 54 (1974), 123-134.

[15] - Stable vector bundles with numerically trivial Chern classes over a hyperelliptic surfaces, Nagoya Math., J., 59 (1975), 107-134.

\section{Nagoya University}

